\title{
Irregularities in the NBS (1955) Provisional Temperature Scale
}

\author{
Hans M. Roder \\ Institute for Materials Research
}

\begin{abstract}
(August 5, 1965)
This paper presents evidence for small irregularities in the temperature scales employed between 50 and $100^{\circ} \mathrm{K}$. The results are derived from the raw data defining the PVT surface of parahydrogen and are given in terms of temperature adjustments to the NBS (1955) scale. The results suggest that an improvement of the temperature scales presently in use would be desirable.
\end{abstract}

Key Words: Gas thermometer, international practical temperature scale, NBS (1955) provisional temperature scale, platinum resistance thermometer.

The purpose of this report is to illustrate several irregularities in the NBS (1955) scale for low temperatures and to estimate their values. In addition, the junction of the NBS (1955) scale with the lower end of the International Practical Temperature Scale (IPTS), near $90^{\circ} \mathrm{K}$, is examined for irregular behavior. The NBS (1955) provisional scale for low temperatures is based on a comparison of platinum resistance thermometers with a helium gas thermometer between 14 and $83{ }^{\circ} \mathrm{K}[1] .{ }^{1}$ The scale is used in the U.S. to calibrate precision resistance thermometers between 10 and $90{ }^{\circ} \mathrm{K}$. Irregularities in the scale are known to exist [2]; however, the irregularities are less than $\pm 0.02^{\circ} \mathrm{K}$, the accuracy claimed for the original experiment. The International Practical Temperature Scale (IPTS) [3] is the working scale adopted by international agreement for use at temperatures above $90^{\circ} \mathrm{K}$. Earlier experiments [see, for instance, ref. 4] show how this scale differs between 90 and $300{ }^{\circ} \mathrm{K}$ from the Kelvin scale. A good summary of all experiments of this type is given by Stimson [5]. It is interesting to note that recent accurate computations of thermodynamic functions take the difference between the IPTS and the Kelvin scale into account [6].

Low level irregularities in temperature scales become troublesome as the accuracy in experiments improves, especially so if the variable measured is expected to vary continuously (smoothly) with absolute temperature. Recent experimental evidence $[2,6$, $7,8]$ suggests that this level of experimental accuracy has been reached. Irregularities in heat capacities are attributed to imperfections in the temperature

1 The present NBS provisional temperature scale is designated NBS (1955) and is defined to be $0.01 \mathrm{deg}$ lower than the scale given in reference [1], found in the referenced literature at the end of this paper. scales employed [9]. These imperfections can also be derived from the PVT surface of a gas, provided that the experimental data are sufficiently accurate, and, further, that the data were taken along lines of constant volume. Given these conditions, each line of constant volume may be treated as an independent gas thermometric experiment where the pressures are obtained as a function of temperature in a closed system, and the assumption is made that for each line of constant volume the values of pressure should vary continuously (smoothly) with absolute temperature.

The results of the present analysis are based on 920 of the 1200 points recently obtained by Goodwin et al., $[10]$ defining the PVT surface of parahydrogen. Table 1 of reference [10] presents the experimental values in terms of lines of nearly constant volume called pseudo-isochores. Each experimental point is considered as an independent determination. Pseudoisochores at high densities which do not include pressure entries at $50{ }^{\circ} \mathrm{K}$ or higher have been omitted from the analysis for reasons to be discussed later. On all other pseudo-isochores the pressure entries below $50{ }^{\circ} \mathrm{K}$ were included in the least squares fitting of the analytical representation, but as explained later these pressures could not be utilized in the analysis. The pseudo-isochores are used rather than the partially smoothed data (lines of constant volume, table 2 of reference [10]) because the smoothing may have introduced an additional error, and because it was desired to retain the weighting in density as given by the experiment.

Ideally, the analysis should be performed by treating the temperature as a function of pressure and obtaining the desired temperature adjustments directly. Lack of a suitable analytical representation for $T=f(P)$ 
forced us to approach the problem indirectly in the four steps given below:

1. Fit each pseudo-isochore with an appropriate analytical representation:

2 . obtain deviations in pressure $\left(P_{\text {calc }}-P_{\text {experimental }}\right)$ for each pseudo-isochore at each temperature;

3 . convert each deviation in pressure to an equivalent deviation in temperature:

4. seek a consistent pattern of temperature deviations by considering all pseudo-isochores together; i.e., temperature scale shifts which would improve the smoothness of the raw data and cause the deviations in pressure to appear random. (For a given temperature the temperature adjustment was established by averaging the equivalent temperature deviations obtained in step 3 for all pseudo-isochores.)

The analytical representation used in step 1 above is of critical importance to the analysis performed. Many of the pseudo-isochores exhibit inflection points, and a few of these occur at temperatures as high as $50{ }^{\circ} \mathrm{K}$. As many as five different analytical representations might be required to define the PVT surface accurately at lower temperatures [11]. The analysis, therefore, was restricted to temperatures above 50 ${ }^{\circ} \mathrm{K}$, using the same analytical representation employed recently in a compilation of thermodynamic functions [12]:

$$
P_{\text {calc }}=A T^{2}+B T+C+D / T+E / T^{2} .
$$

The analysis was also performed by smoothing fourth differences [13], and by a technique which places certain restraints on the derivatives; i.e., a "splinefit" [14], yielding results essentially identical to the ones presented here.

The total deviation in pressure obtained in step 2 above arises from the following contributions:

$E_{1}$ the error made in the actual measurement of pressure;

$E_{2}$ the error made in the experimental determination of temperature on the NBS (1955) scale or on the IPTS;

$E_{3}$ the irregularities in the temperature scale usedNBS (1955) and the lower end of the IPTS scale;

$E_{4}$ the deviation between the Kelvin scale and the scale fashioned by the particular analytical representation used.

In the analysis we attempt to separate the error $E_{3}$ from the others. Maximum values for the error $E_{1}$ have been estimated by Goodwin [15, table 2]. It is pertinent to use the rms combination of his various uncertainties resulting in the following values: 0.00408 atm at a total pressure of $2 \mathrm{~atm}, 0.00435$ at $20 \mathrm{~atm}$, and 0.00830 at $200 \mathrm{~atm}$. The error $E_{2}$ arises from the experimental uncertainty and a certain reproducible error. The uncertainty expresses the ability of the experimenter to maintain a certain desired temperature during data recording and has been estimated to be $0.00025{ }^{\circ} \mathrm{K}[11]$. This uncertainty and the error $E_{1}$ are random; combined, they cause the spread in deviations illustrated in figure 1 . The reproducible

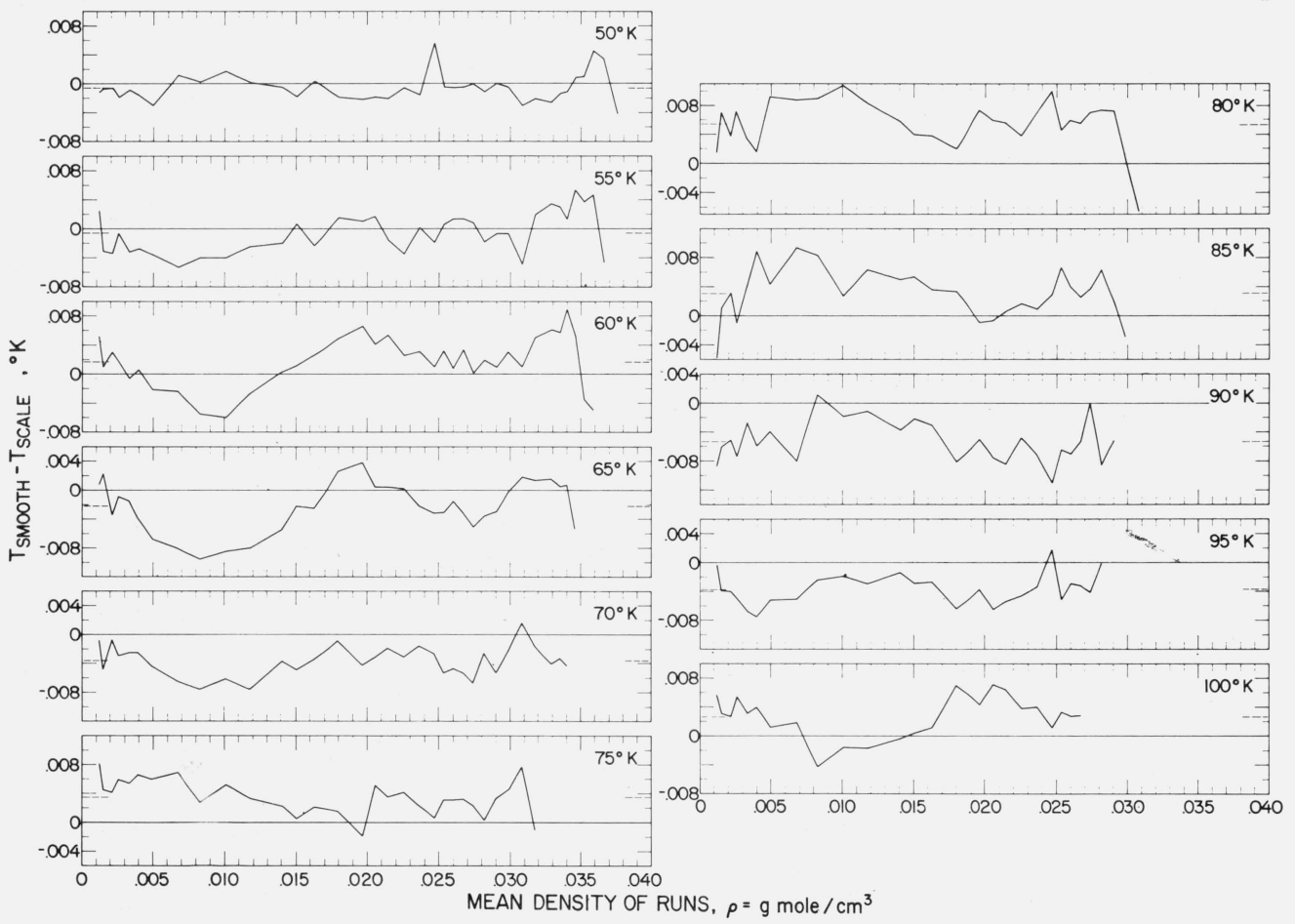

FIGURE 1. The individual temperature deviations.

Equivalent temperature deviations of observed pressures from values calculated using equations fitted to the data (pseudo-isochores). Every junction of the broken straight lines represents the deviation of the observed pseudo-isochore at the temperatures indicated. The results from the same pseudo-isochores are found along vertical lines and those from different pseudo-isochores along horizontal lines 
part of the error $E_{2}$ assumes a given value for each temperature attained in the experiment. It is based on consideration of the various components of the experiment; certain maximum values have been estimated by Goodwin [15, table 3]. By the nature of this analysis the reproducible part of the error $E_{2}$ will be lumped with the error $E_{3}$ which we wish to determine. The error $E_{4}$ has been included to indicate that a constant or slightly varying "offset" in temperature may exist between the smooth analytical representation used and the assumed ideal, but unknown, function of pressure versus thermodynamic temperature. This error also includes the continuously (smoothly) changing "offset" which may be introduced by using pseudo-isochores rather than the true line of constant volume. Results obtained from the other smoothing techniques indicated previously and from fitting the isochores of table 2 in reference [10] permit an estimate of $\pm 0.001{ }^{\circ} \mathrm{K}$ as the maximum "offset" caused by the error $E_{4}$. Any small error made in the assignment of density to a particular pseudo-isochore has been shown to be constant for that pseudo-isochore $[10]$ and will not affect this analysis.

TABiE 1. Results of the Analysis

\begin{tabular}{c|c|c|c}
\hline \hline & $\begin{array}{c}\text { Mean } \\
\text { Temperature } \\
\text { adjustment, } \\
T_{\text {scale }}- \\
T_{\text {smooth }}\end{array}$ & $\begin{array}{c}\text { Standard } \\
\text { error } \\
\text { of } \\
\text { temperature } \\
\text { adjustment }\end{array}$ & $\begin{array}{c}\text { Number of runs } \\
\text { on which } \\
\text { columns 2 } \\
\text { and 3 are } \\
\text { based }\end{array}$ \\
\hline${ }^{\circ} \mathrm{K}$ & ${ }^{\circ} \mathrm{K}$ & ${ }^{\circ} \mathrm{K}$ & \\
50 & 0.0006 & 0.0003 & 38 \\
5.5 & .0006 & .0005 & 37 \\
60 & -.0017 & .0006 & 36 \\
65 & .0022 & .0006 & 34 \\
70 & .0036 & .0004 & 33 \\
75 & -.0035 & .0004 & 30 \\
80 & -.0054 & .0006 & 29 \\
85 & -.0031 & .0007 & 28 \\
90 & .0053 & .0006 & 27 \\
95 & .0037 & .0004 & 26 \\
100 & -.0026 & .0006 & 24
\end{tabular}

The results of the analysis are shown in figure 1 . where the individual equivalent temperature deviations, $T_{\text {smooth }}-T_{\text {scale, }}$, have been plotted, for each temperature considered, as a function of the mean density of the pseudo-isochores (runs). The results are summarized in table 1, where the temperature adjustments shown are the averages of the equivalent temperature deviations for all pseudo-isochores at a given temperature. These averages or mean values are indicated by dashed lines in the ordinates of figure 1 . The standard errors of the mean temperature adjustments are given in column 3 of table 1 and are indicated in figure 2 . The mean temperature adjustments are 2 to 9 times larger than the corresponding standard errors except for $55^{\circ} \mathrm{K}$, so that, based on the number of runs in column 4, table 1 , these mean adjustments are significantly different from zero. The temperature adjustments are presumed to be a good indication of the irregularities in the temperature scales because

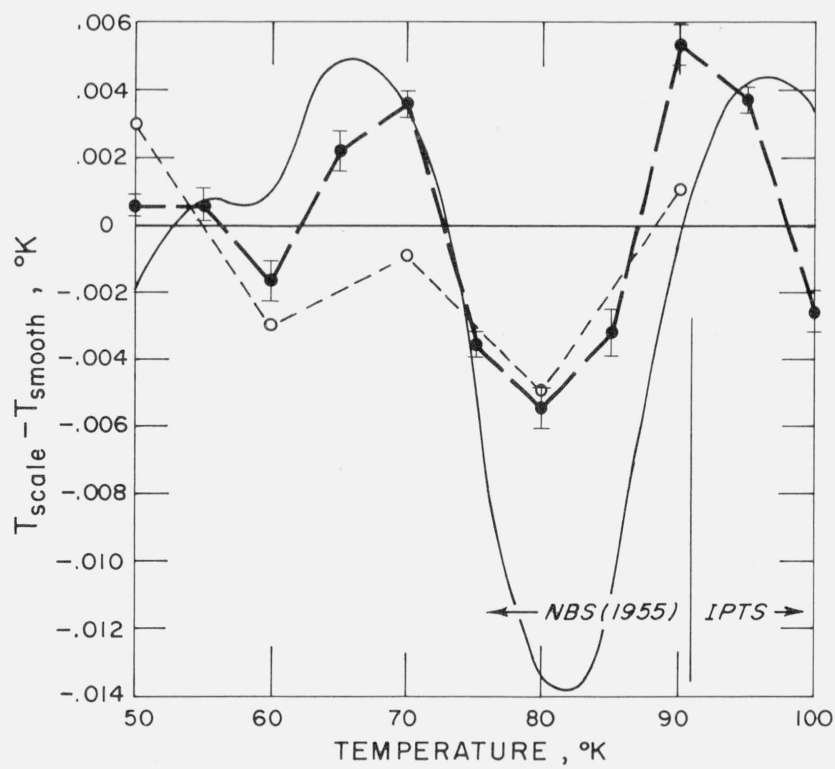

FIGURE 2. The irregularities of the NBS (1955) scale and the IPTS. - - this paper relative to the "smoothed" scale, where I is the standard error of the temperature adjustment - see text. - Barber $[2 \mid$ relative to the average of the four scales considered-see text. - Furukawa and Reilly $|9|$ relative to their smoothed scale.

if they are applied to the raw data the absolute sum of the pressure deviations is reduced from 3.27 atm to $2.16 \mathrm{~atm}$. For comparison we have computed the sum of the errors $E_{1}$ linearly interpolating between the estimates of error established previously. The sum so obtained is $2.30 \mathrm{~atm}$, in excellent agreement with the value 2.16 at $\mathrm{m}$ above.

The temperature adjustments between the NBS (1955) scale or the IPTS and the "smoothed" scale are plotted in figure 2. For ease of comparison the ordinate has been reversed and is now $T_{\text {scale }}-T_{\text {smooth }}$. The resemblance between the present work and the results of Barber [2] and of Furukawa and Reilly [9] is striking. Barber considered the temperature scales established at the National Physical Laboratory [2], at the National Bureau of Standards [1], at the Pennsylvania State University [16], and at the Physicotechnical, Radio-technical Measurements Institute [17]. He compared these scales on common terms by using a "best" value of the oxygen point on the thermodynamic scale, and by adjusting them to the same virial coefficients and to the same expansion data for copper. The deviation of the NBS scale from the mean of the four scales considered by Barber is also plotted in figure 2. The resemblance to the work of Furukawa and Reilly is perhaps superficial. However, their process permits the establishment of a uniform degree from 20 to $380{ }^{\circ} \mathrm{K}$ while keeping the average size of the degree in this interval the same as for NBS (1955)IPTS. 
The results given in this paper illustrate certain irregularities in the temperature scales employed between 50 and $100{ }^{\circ} \mathrm{K}$. They indicate that the NBS (1955) scale is slightly irregular and does not join smoothly to the lower end of the IPTS scale. The adjustments obtained for the lower end of the IPTS scale are qualitatively in agreement with earlier experiments [4] and with the evaluation based on the heat capacity data [9]. Thus, the deviation pattern established from the PVT surface of parahydrogen reinforces earlier work and suggests that an improvement in the temperature scales would be desirable.

The author is indebted to R. J. Corruccini, R. D. Goodwin, L. A. Weber, and B. A. Younglove for many valuable comments and discussions.

\section{References}

[1] H. J. Hoge and F. G. Brickwedde. Establishment of a temperature scale for the calibration of thermometers between 14 and $83^{\circ} \mathrm{K}$, J. Res. NBS 22, 351 (March 1939).

[2] C. R. Barber, The establishment of a practical scale of temperature for the range $10-90{ }^{\circ} \mathrm{K}$, Brit. J. Appl. Phys. 13, 235 (May 1962):

[3] H. F. Stimson, International practical temperature scale of 1948. Text revision of 1960, J. Res. NBS 65A (Phys. and Chem.) No. 3, 139 (May 1961).

[4] W. H. Keesom and B. G. Dammers, Comparison of some platinum thermometers with the helium thermometer between 0 and $-183{ }^{\circ} \mathrm{C}$, Physica 2, 1080 (1935).

[5] H. F. Stimson, Heat units and temperature scales for calorimetry, American Journal of Physics 23, 614 (Dec. 1955).

[6] W. de Graaff, Compressibility isotherms and thermodynamic functions of hydrogen and deuterium. Conclusions regarding the intermolecular field, Thesis, University of Amsterdam, 29 June 1960, page 13.

[7] G. T. Armstrong, Calorimetry, Physics Today 17, 50 (Feb. 1964).
[8] G. T. Furukawa, M. L. Reilly, and J. H. Piccirelli, Calorimetric properties of some alkali pentaborate hydrates from 15 to $370{ }^{\circ}$ K, J. Res. NBS 68A (Phys. and Chem.) No. 4, 381 (July 1964).

[9] G. T. Furukawa and M. L. Reilly, Application of precise heatcapacity data to the analysis of the temperature intervals of the NBS (1955) and the International Practical Temperature Scales in the region of $90^{\circ} \mathrm{K}$. J. Res. NBS 69A (Phys. and Chem.) No. 1, 5 (Jan. 1965).

[10] R. D. Goodwin, D. E. Diller, H. M. Roder, and L. A. Weber, Pressure-density-temperature relations of fluid para-hydrogen from $15{ }^{\circ} \mathrm{K}$ to $100^{\circ} \mathrm{K}$ at pressures to 350 at mospheres, J. Res. NBS 67A (Phys. and Chem.) No. 2, 173 (March 1963).

[11] R. D. Goodwin, private communication.

[12 H. M. Roder, L. A. Weber, and R. D. Goodwin, Thermodynamic and related properties of parahydrogen from the triple point to $100^{\circ} \mathrm{K}$ at pressures to $340 \mathrm{~atm}$., NBS Monograph 94 (1965).

[13] C. Lanczos, Applied Analysis, pp. 316-320 (Prentice Hall, Englewood Cliffs, N.J., 1956).

[14] F. Landis and E. N. Nielson, The determination of thermodynamic properties by direct differentiation techniques, Progress in International Research on Thermodynamic and Transport Properties, Second Symposium on Thermophysical Properties, pp. 218-227 (Academic Press, New York, N.Y. 1962).

[15] R. D. Goodwin, Apparatus for determination of pressuredensity-temperature relations and specific heats of hydrogen to 350 atmospheres at temperatures above $14{ }^{\circ} \mathrm{K}$, J. Res. NBS 65 C (Eng. and Instr.) No. 4, 231 (Oct. 1961).

[16] G. W. Moessen, J. G. Aston, and R. G. Ascah, The Pennsylvania State University thermodynamic temperature scale below $90{ }^{\circ} \mathrm{K}$ and the normal boiling points of oxygen and normal hydrogen on the thermodynamic scale, Temperature, Its Measurement and Control in Science and Industry, Vol. 3, pt 1, p. 91 (Reinhold Publishing Corp., New York, N.Y., 1962).

[17] A. C. Borovick-Romanov, P. G. Strelkov, M. P. Orlova, and D. N. Astrov, The I.M.P.R. temperature scale for the 10 to $90{ }^{\circ} \mathrm{K}$ region. Temperature, Its Measurement and Control in Science and Industry, Vol. 3, pt 1, p. 113 (Reinhold Publishing Corp., New York, N.Y., 1962).

(Paper 69A6-374) 